Decomposing one-relator products of cyclic groups into free products with amalgamation

This article has been downloaded from IOPscience. Please scroll down to see the full text article.

1998 Sb. Math. 1891125

(http://iopscience.iop.org/1064-5616/189/8/A02)

View the table of contents for this issue, or go to the journal homepage for more

Download details:

IP Address: 87.252.227.105

The article was downloaded on 01/03/2011 at 14:41

Please note that terms and conditions apply. 


\title{
Decomposing one-relator products of cyclic groups into free products with amalgamation
}

\author{
V. V. Benyash-Krivets
}

\begin{abstract}
The problem of the decomposition of one-relator products of cyclics into non-trivial free products with amalgamation is considered. Two theorems are proved, one of which is as follows.

Let $G=\left\langle a, b \mid a^{2 n}=R^{m}(a, b)=1\right\rangle$, where $n \geqslant 0, m \geqslant 2$, and $R(a, b)$ is a cyclically reduced word containing $b$ in the free group on $a$ and $b$. Then $G$ is a non-trivial free product with amalgamation.
\end{abstract}

One consequence of this theorem is a proof of the conjecture of Fine, Levin, and Rosenberger that each two-generator one-relator group with torsion is a non-trivial free product with amalgamation.

Bibliography: 13 titles.

\section{Introduction}

A one-relator free product of a family of groups $\left\{G_{i}\right\}, i \in I$, is the group $G=\left(* G_{i}\right) / N(S)$, where $S$ is a cyclically reduced word in the free product $* G_{i}$ and $N(S)$ is its normal closure. We call $S$ a relator. One-relator free products share many properties with one-relator groups [1]. We consider the case when the $G_{i}$ are cyclic groups and the relator is a proper power, that is, $S=R^{m}$, where $R$ is a cyclically reduced word in $* G_{i}$ and $m \geqslant 2$.

Definition 1. A group $G$ having a presentation

$$
G=\left\langle a_{1}, \ldots, a_{n} \mid a_{1}^{l_{1}}=\cdots=a_{n}^{l_{n}}=R^{m}\left(a_{1}, \ldots, a_{n}\right)=1\right\rangle,
$$

where $n \geqslant 2, m \geqslant 2, l_{i}=0$ or $l_{i} \geqslant 2$ for all $i$, and $R\left(a_{1}, \ldots, a_{n}\right)$ is a cyclically reduced word in the free group on $a_{1}, \ldots, a_{n}$, is called a one-relator product of $n$ cyclics.

This paper considers the problem of the decomposition of one-relator products of cyclics into non-trivial free products with amalgamation. The first general results on the decomposition of such groups were obtained in [2]. Theorem 3 in [2] says

This research was carried out with the financial support of the Institute of Mathematics of the National Academy of Sciences of Belarus in the framework of the National Programme of Fundamental Research "Mathematical Structures".

AMS 1991 Mathematics Subject Classification. Primary 20 E06. 
that if $G$ is a free one-relator product of $n$ cyclics $(n \geqslant 3)$, then $G$ is a non-trivial free product with amalgamation.

The case of free one-relator products of 2 cyclics, that is, of groups

$$
G=\left\langle a, b \mid a^{m}=b^{n}=R^{l}(a, b)=1\right\rangle
$$

where $l \geqslant 2$ and $R(a, b)$ is a cyclically reduced word in the free group on $a$ and $b$, is considerably more complicated. Such groups are called generalized triangle groups. Sometimes these groups admit a decomposition into a non-trivial free product with amalgamation and sometimes they do not. For example, it is known (cf. [3]) that the ordinary triangle groups

$$
T(m, n, p)=\left\langle a, b \mid a^{m}=b^{n}=(a b)^{p}=1\right\rangle
$$

where $m, n, p \geqslant 2$, are non-trivial free products with amalgamation. Zieschang [3] has studied the problem of the decomposition into a non-trivial free product with amalgamation for planar discontinuous groups. He has given a complete answer to the question when such a group $G$ is a non-trivial free product with amalgamation in all cases except for the groups

$$
H_{1}=\left\langle a, b \mid[a, b]^{n}=1\right\rangle \text { and } H_{2}=\left\langle c, d \mid c^{2}=[c, d]^{n}=1\right\rangle, n \geqslant 2 .
$$

Rosenberger [4] has proved that the groups $H_{1}$ and $H_{2}$ are non-trivial free products with amalgamation if $n$ is not a power of 2 . In recent papers [5] and [6] this property was established for arbitrary $n$. This result is also an immediate consequence of Theorem 1. The following conjecture was stated in [2].

Conjecture 1. A two-generator one-relator group with torsion is a non-trivial free product with amalgamation.

Note that if a group $G$ has more that 2 generators, then the assertion of Conjecture 1 holds by Theorem 3 in [2] mentioned above. Moreover, this conjecture has been proved in [2] for groups $G$ having a presentation $G=\left\langle a, b \mid R^{m}(a, b)=1\right\rangle$, where $m \geqslant 2$ and $R(a, b)$ is a cyclically reduced word not belonging to the derived subgroup of the free group on $a$ and $b$.

In the present paper we prove the following two results.

Theorem 1. Let $G=\left\langle a, b \mid a^{2 n}=R^{m}(a, b)=1\right\rangle$, where $n \geqslant 0, m \geqslant 2$, and $R(a, b)$ is a cyclically reduced word containing $b$ in the free group on $a$ and $b$. Then $G$ is $a$ non-trivial free product with amalgamation.

Theorem 2. Let $G=\left\langle a, b \mid a^{n}=R^{m}(a, b)=1\right\rangle$, where $n=0$ or $n \geqslant 2, m \geqslant 3$, and $R(a, b)$ is a cyclically reduced word containing $b$ in the free group on $a$ and $b$. If $R(a, b)=a^{u_{1}} b^{v_{1}} \cdots a^{u_{s}} b^{v_{s}}$, where $0<u_{i}<n, v_{i} \neq 0$ for $i=1, \ldots, s$, and $\prod_{i=1}^{s}\left|v_{i}\right| \geqslant 3$, then $G$ is a non-trivial free product with amalgamation. 
Corollary 1. Let $G$ be a two-generator one-relator group with torsion. Then $G$ is a non-trivial free product with amalgamation.

Proof of Corollary 1. It is well known (see, for example, [7]) that if $G$ is a finitely generated one-relator group with torsion then $G$ has a presentation of the form $G=\left\langle a_{1}, \ldots, a_{n} \mid R^{m}\left(a_{1}, \ldots, a_{n}\right)=1\right\rangle$, where $m \geqslant 2$ and $R\left(a_{1}, \ldots, a_{n}\right)$ is a cyclically reduced word in the free group on $a_{1}, \ldots, a_{n}$. In our case $G=$ $\left\langle a, b \mid R^{m}(a, b)=1\right\rangle$, so that $G$ is a non-trivial free product with amalgamation by Theorem 1 .

Corollary 2. The groups $H_{1}=\left\langle a, b \mid[a, b]^{n}=1\right\rangle$ and $H_{2}=\left\langle c, d \mid c^{2}=[c, d]^{n}=1\right\rangle$ are non-trivial free products with amalgamation for each $n \geqslant 2$.

\section{$\S 1$. Some auxiliary results}

Here we shall prove several auxiliary results. Throughout we shall denote the identity matrix in $\mathrm{SL}_{2}(\mathbb{C})$ by $E$, the ring of algebraic integers in $\mathbb{C}$ by $\mathcal{O}$, the free group of rank 2 with generators $g$ and $h$ by $F_{2}=\langle g, h\rangle$, and the trace of a matrix $X$ by $\operatorname{tr} X$. The following result of Bass [8] plays a key role in the proof of Theorems 1 and 2 .

Proposition 1 (see $[8])$. Let $H$ be a finitely generated subgroup of $\mathrm{GL}_{2}(\mathbb{C})$. Then one of the following cases must occur:

(1) there exists an epimorphism $f: H \rightarrow \mathbb{Z}$ such that $f(u)=0$ for all unipotent

(2) $H$ is conjugate to a subgroup of the group of triangular matrices $\left(\begin{array}{ll}a & b \\ 0 & c\end{array}\right)$
with roots of unity a and $c$;

(3) $H$ is conjugate to a subgroup of the group $\mathrm{GL}_{2}(\mathcal{O})$;

(4) $H$ is a non-trivial free product with amalgamation.

The following observation is useful in the construction of matrices of finite order in $\mathrm{SL}_{2}(\mathbb{C})$.

Lemma 1. Let $m>1$ and let $X \in \mathrm{SL}_{2}(\mathbb{C})$. If $\operatorname{tr} X= \pm 2 \cos (r \pi / m)$, where $r \in\{1, \ldots, m-1\}$, then $X^{2 m}=E$. In particular, if $\operatorname{tr} X=0$, then $X^{2}=-E$.

Proof. Let, for example, $\operatorname{tr} X=2 \cos (r \pi / m)$. Then the characteristic polynomial of $X$ has the roots $\alpha=\cos (r \pi / m)+i \sin (r \pi / m)$ and $\alpha^{-1}=\cos (r \pi / m)-i \sin (r \pi / m)$, where $\alpha$ is some $2 m$ th root of unity. Consequently, $X$ is conjugate to the matrix $X_{1}=\operatorname{diag}\left(\alpha, \alpha^{-1}\right)$ and therefore $X^{2 m}=E$.

In what follows we shall require so-called 'Fricke characters' (see [9]-[11]). For each $w=w(g, h) \in F_{2}$ one can consider the following regular function:

$$
\tau_{w}: \mathrm{SL}_{2}(\mathbb{C}) \times \mathrm{SL}_{2}(\mathbb{C}) \rightarrow \mathbb{C}, \quad \tau_{w}(A, B)=\operatorname{tr}(w(A, B)) .
$$

It has been proved in [9] (see also [12]) that for each $w \in F_{2}$ we have

$$
\tau_{w}=Q_{w}\left(\tau_{g}, \tau_{h}, \tau_{g h}\right)
$$


where $Q_{w} \in \mathbb{Z}[x, y, z]$ is a polynomial with integral coefficients. The function $\tau_{w}$ is called a Fricke character and the polynomial $Q_{w}$ is the Fricke polynomial of the element $w \in F_{2}$. Let $u$ and $v$ be arbitrary elements of $F_{2}$. The following relations for Fricke characters follow from the corresponding relations between the traces of arbitrary matrices in $\mathrm{SL}_{2}(\mathbb{C})$ and can be readily verified:
(1) $\tau_{u^{-1}}=\tau_{u}$;
(2) $\tau_{u v}=\tau_{v u}$;
(3) $\tau_{v u v^{-1}}=\tau_{u}$;
(4) $\tau_{u v}=\tau_{u} \tau_{v}-\tau_{u v^{-1}}$

The following assertion is well known and can be easily proved by straightforward computations, although it is difficult to give an explicit reference.

Lemma 2. For all $\alpha, \beta, \gamma \in \mathbb{C}$ there exist matrices $A, B \in \mathrm{SL}_{2}(\mathbb{C})$ such that

$$
\tau_{g}(A, B)=\operatorname{tr} A=\alpha, \quad \tau_{h}(A, B)=\operatorname{tr} B=\beta, \quad \text { and } \quad \tau_{g h}(A, B)=\operatorname{tr} A B=\gamma .
$$

In particular, this lemma means that the Fricke characters $\tau_{g}, \tau_{h}$, and $\tau_{g h}$ are algebraically independent over $\mathbb{C}$, and therefore the Fricke polynomial $Q_{w}$ of an element $w$ is well defined. Next, we require an explicit formula for the Fricke polynomial obtained in [13]. To formulate this result we consider polynomials $P_{n}(\lambda)$ satisfying the recurrence relations

$$
P_{n}(\lambda)=\lambda P_{n-1}(\lambda)-P_{n-2}(\lambda)
$$

and the initial conditions

$$
P_{0}(\lambda)=1, \quad P_{-1}(\lambda)=0 .
$$

If $n<0$, then we set

$$
P_{n}(\lambda)=-P_{|n|-2}(\lambda)
$$

The degree of the polynomial $P_{n}(\lambda)$ is equal to $n$ if $n>0$ and to $|n|-2$ if $n<0$.

Lemma 3. (1) The polynomial $P_{n}(\lambda), n \geqslant 1$, has $n$ zeros, described by the formula

$$
\lambda_{n, k}=2 \cos \frac{k \pi}{n+1}, \quad k=1,2, \ldots, n .
$$

(2) $P_{n-1}(2)=n$ for each $n \in \mathbb{Z}$.

(3) Let $x=\tau_{g}$, let $y=\tau_{h}$, and let $z=\tau_{g h}$. Then

$$
Q_{g h^{n}}(x, y, z)=P_{n-1}(y) z-P_{n-2}(y) x .
$$

Proof. (1) It is easy to verify by induction on $n$ that

$$
P_{n}(2 \cos \varphi)=\frac{\sin (n+1) \varphi}{\sin \varphi} .
$$

Assertion (1) can now be obtained by a straightforward computation.

Assertions (2) and (3) can be proved by induction on $n$ (as regards (2), see also [12], formula (6)).

Further, let $w=g^{\alpha_{1}} h^{\beta_{1}} \cdots g^{\alpha_{s}} h^{\beta_{s}} \in F_{2}$ be a cyclically reduced word in $F_{2}$, and let $x=\tau_{g}, y=\tau_{h}$, and $z=\tau_{g h}$. We treat the Fricke polynomial $Q_{w}(x, y, z)$ as a polynomial in $z$. Let

$$
Q_{w}(x, y, z)=M_{n}(x, y) z^{n}+M_{n-1}(x, y) z^{n-1}+\cdots+M_{0}(x, y) .
$$


Proposition 2 (see [13]). The degree of the Fricke polynomial $Q_{w}(x, y, z)$ with respect to $z$ is equal to $s$, that is, the number of blocks of the form $g^{\alpha_{i}} h^{\beta_{i}}$ in $w$. The leading coefficient $M_{s}(x, y)$ of the polynomial $Q_{w}(x, y, z)$ has the following form:

$$
M_{s}(x, y)=\prod_{i=1}^{s} P_{\alpha_{i}-1}(x) P_{\beta_{i}-1}(y) .
$$

The following lemma plays an important role in the proof of Theorems 1 and 2 .

Lemma 4. Let $G=\left\langle a, b \mid a^{n}=R^{m}(a, b)=1\right\rangle$ and let $A, B \in \mathrm{SL}_{2}(\mathbb{C})$ be matrices such that $\operatorname{tr} A=\alpha$, where $\alpha= \pm 2 \cos (t \pi / n)$ for some $t \in\{1, \ldots, n-1\}$, and $Q_{R}(\alpha, y, z)=c$, where $Q_{R}$ is the Fricke polynomial of the element $R(g, h) \in F_{2}$, $c= \pm 2 \cos (r \pi / m)$ for some $r \in\{1, \ldots, m-1\}, y=\operatorname{tr} B$, and $z=\operatorname{tr} A B$. Let $H=\langle A, B\rangle$. Assume that two following conditions are satisfied:

(1) there exists a unipotent (or finite-order) element $u=A^{\alpha_{1}} B^{\beta_{1}} \cdots A^{\alpha_{s}} B^{\beta_{s}}$ in $H$ such that $l=\sum_{i=1}^{s} \beta_{i} \neq 0$;

(2) there exists an element $h \in H$ such that $\operatorname{tr} h \notin \mathcal{O}$.

Then the group $G$ is a non-trivial free product with amalgamation.

Proof. We claim that the group $H$ fails conditions (1)-(3) of Proposition 1. Let $f: H \rightarrow \mathbb{Z}$ be an epimorphism such that $f(z)=0$ for all unipotent elements $z \in H$. Then $f(A)=0$ because $A^{2 n}=E$ by Lemma 1. Further, $f(u)=l f(B)=0$, therefore $f(B)=0$ because, by assumption, $u$ is either unipotent or of finite order. Hence $f(H)=\{0\}$, which is a contradiction. Next, there exists by assumption an element $h \in H$ such that $\operatorname{tr} h \notin \mathcal{O}$. Hence $H$ does not satisfy conditions (2) and (3) of Proposition 1 and $H$ is a non-trivial free product with amalgamation, that is, $H=H_{1} *_{F} H_{2}$ with $H_{1} \neq F \neq H_{2}$. Since $-E \in Z(H)$, it follows that $-E \in F$. Let $\bar{A}, \bar{B}, \bar{H}, \bar{H}_{1}, \bar{H}_{2}$, and $\bar{F}$ be the images of $A, B, H, H_{1}, H_{2}$, and $F$ in $\mathrm{PSL}_{2}(\mathbb{C})$, respectively. Then $\bar{H}_{1} \neq \bar{F} \neq \bar{H}_{2}$, and therefore $\bar{H}=\bar{H}_{1} * \bar{F}_{2} \bar{H}_{2}$ is a nontrivial free product with amalgamation. The condition $Q_{R}(\alpha, y, z)=c$ means that $\operatorname{tr} R(A, B)=c$, so that $R^{2 m}(A, B)=E$ by Lemma 1 . Thus, $\bar{A}^{n}=R^{m}(\bar{A}, \bar{B})=1$ in $\mathrm{PSL}_{2}(\mathbb{C})$, so that $\bar{H}$ is an epimorphic image of $G$ and we obtain the assertion of Lemma 4. (It is well known that if $\varphi: \Gamma_{1} \rightarrow \Gamma_{2}$ is an epimorphism of groups and $\Gamma_{2}$ is a non-trivial free product with amalgamation, then so also is $\Gamma_{1}$.)

The following lemma will be repeatedly used in what follows.

Lemma 5. (1) For $s, m, M \in \mathbb{Z}$ satisfying the conditions $m \geqslant 2$ and $|M| \geqslant 3$ there exist $\varepsilon \in\{-1,1\}$ and $r \in\{1, \ldots, m-1\}$ such that $\left((-1)^{s} 2-c\right) / M \notin \mathcal{O}$, where $c=2 \varepsilon \cos (r \pi / m)$.

(2) For $m \in \mathbb{Z}, m \geqslant 2$, there exists an integer $r \in\{1, \ldots, m-1\}$ such that $(2+2 \cos (r \pi / m))^{-1} \notin \mathcal{O}$.

(3) If $m \in \mathbb{Z}, m \geqslant 3$, then $\cos (\pi / m) \notin \mathcal{O}$.

(4) For all $m, M \in \mathbb{Z}, m \geqslant 3$ and $|M| \geqslant 3$, there exists $r \in\{1, \ldots, m-1\}$ such that $(4 / M) \cos (r \pi / m) \notin \mathcal{O}$.

Proof. (1) Without loss of generality we can assume that $s$ is even. Assume also that for all $\varepsilon \in\{-1,1\}$ and $r \in\{1, \ldots, m-1\}$ the ratio $(2-c) / M$ is an algebraic integer. Then $(2-c) / M+(2+c) / M=4 / M$ is an algebraic integer too. 
Since $|M| \geqslant 3$, this is possible only if $|M|=4$. For definiteness, let $M=4$. If $m$ is even, then we set $r=m / 2$. In this case $c=0$ and $2 / M=\frac{1}{2} \notin \mathcal{O}$ which is a contradiction. Assume that $m$ is odd and $F_{1}=\{2 \cos (2 r \pi / m) \mid r=1, \ldots, m-1\}$. Then

$$
\sum_{c \in F_{1}} \frac{2-c}{4}=\frac{2(m-1)-\sum_{c \in F_{1}} c}{4}=\frac{2(m-1)+1}{4},
$$

because $1+\sum_{c \in F_{1}} c=0$ as the sum of the $m$ th roots of unity. Obviously, $(2(m-1)+1) / 4$ is not an algebraic integer; this is a contradiction proving assertion (1).

(2) Since

$$
\frac{1}{2+2 \cos (r \pi / m)}=\frac{1}{4 \cos ^{2}(r \pi /(2 m))},
$$

it is sufficient to prove that $(2 \cos (r \pi /(2 m)))^{-1}$ does not belong to $\mathcal{O}$ for some $r \in\{1, \ldots, m-1\}$. By virtue of Lemma 3 the polynomial $P_{2 m-1}(\lambda)$ has zeros at 0 and $\pm 2 \cos (r \pi /(2 m)), r=1, \ldots, m-1$. It is easy to verify by induction on $m$ that

$$
P_{2 m-1}(\lambda)=\lambda\left(\lambda^{2 m-2}+a_{1} \lambda^{2 m-4}+\cdots+(-1)^{m+1} m\right) .
$$

Hence the numbers $( \pm 2 \cos (r \pi /(2 m)))^{-1}$ are the zeros of $P_{2 m-1}(1 / \lambda)$ or, equivalently, of the polynomial

$$
g_{1}(\lambda)=\lambda^{2 m-2}+\cdots+(-1)^{m+1} \frac{1}{m} .
$$

Since $1 / m \notin \mathcal{O}$, at least one of the zeros of $g_{1}(\lambda)$ is not an algebraic integer and (2) is proved.

(3) It is sufficient to prove that there exists $r \in\{1, \ldots, m-1\}$ such that $\cos (r \pi / m) \notin \mathcal{O}$ because if $\cos (\pi / m) \in \mathcal{O}$, then $\cos (r \pi / m) \in \mathcal{O}$ for each $r \in \mathbb{Z}$. By Lemma 3 the numbers $2 \cos (r \pi / m), r=1, \ldots, m-1$, are the zeros of the polynomial $P_{m-1}(\lambda)$. If $m=2 k$, then it follows from (3) that the numbers $\cos (r \pi /(2 k))$, $r=1, \ldots, 2 k-1$, are the zeros of $g_{1}(2 \lambda)$ or, equivalently, the roots of the polynomial equation

$$
\lambda^{2 k-1}+\cdots+(-1)^{k-1} \frac{k}{2^{2 k-2}} \lambda=0 .
$$

Since $k \geqslant 2$, it follows that $k<2^{2 k-2}$, so that $k / 2^{2 k-2}$ is not an algebraic integer. Hence there exists $r$ such that $\cos (r \pi /(2 k)) \notin \mathcal{O}$.

Let $m=2 k+1$. Then it is easy to verify by induction that

$$
P_{2 k}(\lambda)=\lambda^{2 k}+\cdots+(-1)^{k} .
$$

By Lemma 3 the numbers $\cos (r \pi /(2 k+1)), r=1, \ldots, 2 k$, are the zeros of $P_{2 k}(2 \lambda)$ or, equivalently, the roots of the equation

$$
\lambda^{2 k}+\cdots+\frac{(-1)^{k}}{2^{2 k}}=0 .
$$

Since $(-1)^{k} / 2^{2 k} \notin \mathcal{O}$, there exists $r$ such that $\cos (r \pi /(2 k+1)) \notin \mathcal{O}$. 
(4) It follows from Lemma 3 that the numbers $(4 / M) \cos (r \pi / m), r=1, \ldots, m-1$, are the zeros of $P_{m-1}(M \lambda / 2)$. We consider now two cases.

Let $m=2 k+1$. Then the numbers $(4 / M) \cos (r \pi /(2 k+1)), r=1, \ldots, 2 k$, are by (4) the roots of the equation

$$
\lambda^{2 k}+\cdots+(-1)^{k}\left(\frac{2}{M}\right)^{2 k}=0 .
$$

Since $|M| \geqslant 3$, it follows that $(-1)^{k}(2 / M)^{2 k} \notin \mathcal{O}$, and therefore the last equation also has a root not belonging to $\mathcal{O}$.

Let $m=2 k$. Then the numbers $(4 / M) \cos (r \pi / m), r=1, \ldots, m-1$, are by (3) the roots of the equation

$$
\lambda^{2 k-1}+\cdots+(-1)^{k+1} k\left(\frac{2}{M}\right)^{2 k-2} \lambda=0 .
$$

It is easy to see that $(-1)^{k+1} k(2 / M)^{2 k-2} \notin \mathcal{O}$. This proves assertion (4) and, at the same time, Lemma 5 .

\section{$\S 2$. Proof of Theorem 1}

Let $G$ be a group satisfying the assumptions of Theorem 1 . If $n=0$ or $n>1$, then we consider the group $G_{1}=\left\langle a, b \mid a^{2}=R^{m}(a, b)=1\right\rangle$, which is an epimorphic image of $G$. Thus, we can assume without loss of generality that $n=1$. Our aim is to construct a representation $\varphi: G \rightarrow \mathrm{PSL}_{2}(\mathbb{C})$ such that the group $\varphi(G)$ is a non-trivial free product with amalgamation. Three cases can occur; we shall consider them separately:

(1) $R(a, b)=a b^{n_{1}} \cdots a b^{n_{s}}$ is a cyclically reduced word lying in the subgroup of the free group on $a$ and $b$ generated by $a^{2}$ and the derived subgroup; moreover, there exists $i$ such that $\left|n_{i}\right|>1$;

(2) $R(a, b)$ is as in case 1 , but $\left|n_{i}\right|=1$ for all $i=1, \ldots, s$;

(3) $R(a, b)$ is a cyclically reduced word that does not belong to the subgroup of the free group on $a$ and $b$ generated by $a^{2}$ and the derived subgroup.

Case 1. Let $R(a, b)=a b^{n_{1}} \cdots a b^{n_{s}}$. It follows from our assumptions about the element $R(a, b)$ that $s$ is even and $\sum_{i=1}^{s} n_{i}=0$. To apply Lemma 4 we shall show that there exist matrices $A, B \in \mathrm{SL}_{2}(\mathbb{C})$ such that

$$
\operatorname{tr} A=0, \quad \operatorname{tr} B=2, \quad \text { and } \quad \operatorname{tr} A B=z,
$$

where $z$ is a complex number not belonging to $\mathcal{O}$ and satisfying the equation

$$
Q_{R}(0,2, z)=c,
$$

where $c= \pm 2 \cos (r \pi / m)$ for some $r \in\{1, \ldots, m-1\}$.

By Proposition 2 we can write (5) as follows:

$$
M_{s}(0,2) z^{s}+M_{s-1}(0,2) z^{s-1}+\cdots+M_{0}(0,2)-c=0,
$$

where $M_{s}(0,2)=\prod_{i=1}^{s} P_{0}(0) P_{n_{i}-1}(2)=\prod_{i=1}^{s} n_{i}$ by Lemma 3 . Since $s$ is even, $\sum_{i=1}^{s} n_{i}=0$ and there exists $i$ such that $\left|n_{i}\right|>1$, it follows that $\prod_{i=1}^{s}\left|n_{i}\right| \geqslant 3$. Thus, $\left|M_{s}(0,2)\right| \geqslant 3$. Furthermore, we have the following result. 
Lemma 6. Let $Q_{R}(x, y, z)=M_{s}(x, y) z^{s}+\cdots+M_{0}(x, y)$ be the Fricke polynomial of $R=g h^{n_{1}} \cdots g h^{n_{s}} \in F_{2}$, where $s$ is even, $x=\tau_{g}, y=\tau_{h}$, and $z=\tau_{g h}$. Then $M_{0}(0,2)=(-1)^{s / 2} 2$.

Proof. First, we make the following observations.

(1) $Q_{h^{i}}(0,2, z)=2$, for if $A, B \in \mathrm{SL}_{2}(\mathbb{C})$ and $B$ is a unipotent matrix, then $B^{i}$ is unipotent for any $i$. Hence $\tau_{h^{i}}(A, B)=\operatorname{tr} B^{i}=2$.

(2) It follows from Lemma 3 that $Q_{g h^{i}}(0,2, z)=P_{i-1}(2) z=i z$.

We shall prove the lemma by induction on $s$. If $s=2$, then, using relations (1), we obtain

$$
Q_{g h^{n_{1}} g h^{n_{2}}}(0,2, z)=Q_{g h^{n_{1}}}(0,2, z) Q_{g h^{n_{2}}}(0,2, z)-Q_{h^{n_{2}-n_{1}}}(0,2, z)=n_{1} n_{2} z^{2}-2,
$$

that is, $M_{0}(0,2)=-2$. For arbitrary $s>2$ we have

$$
\begin{aligned}
Q_{R}(0,2, z)=Q_{g h^{n_{1}}}(0,2, z) & Q_{g h^{n_{2} \ldots g h^{n_{s}}}}(0,2, z) \\
& -Q_{g h^{n_{3}} \ldots g h^{n_{s}-n_{1}+n_{2}}}(0,2, z)=n_{1} z f(z)-g(z),
\end{aligned}
$$

where

$$
\begin{aligned}
& f(z)=Q_{g h^{n_{2} \ldots g h^{n_{s}}}}(0,2, z), \\
& g(z)=Q_{g h^{n_{3}} \cdots g h^{n_{s}-n_{1}+n_{2}}}(0,2, z) .
\end{aligned}
$$

We see that the polynomial $Q_{R}(0,2, z)$ has the same constant term as $g(z)$ and, by induction,

$$
M_{0}(0,2)=-(-1)^{(s-2) / 2} 2=(-1)^{s / 2} 2 .
$$

This proves Lemma 6 .

Bearing in mind Lemma 6 we can write (6) in the following form:

$$
z^{s}+\frac{M_{s-1}(0,2)}{M_{s}(0,2)} z^{s-1}+\cdots+\frac{(-1)^{s / 2} 2-c}{M_{s}(0,2)}=0 .
$$

It follows from Lemma 5(1) that one can choose $c= \pm 2 \cos (r \pi / m)$ in the equation (7) such that the constant term $\left((-1)^{s / 2}-c\right) / M_{s}(0,2)$ does not belong to $\mathcal{O}$. Then $(7)$ has a root $z_{0}$ that is not an algebraic integer. By Lemma 2 there exist matrices $A, B \in \mathrm{SL}_{2}(\mathbb{C})$ such that

$$
\operatorname{tr} A=0, \quad \operatorname{tr} B=2, \quad \operatorname{tr} A B=z_{0} .
$$

Lemma 4 completes the proof of Theorem 1 in the first case.

Case 2. Let $R(a, b)=a b^{\varepsilon_{1}} a b^{\varepsilon_{2}} \cdots a b^{\varepsilon_{s}}$. It follows from our assumptions that $s$ is even, $\varepsilon_{i} \in\{-1,1\}$ for $i=1, \ldots, s$, and $\sum_{i=1}^{s} \varepsilon_{i}=0$.

Assume for the moment that $\varepsilon_{j}=\varepsilon_{j+1}$ for some $j<s$. Let $c=a$ and $d=a b^{\varepsilon_{j}}$ be new generators of the group $G$. Then it is not hard to verify that $G$ has a presentation of the following form:

$$
G=\left\langle c, d \mid c^{2}=R_{1}^{m}(c, d)=1\right\rangle,
$$


where $R_{1}(c, d)=c d^{l_{1}} \cdots c d^{l_{t}}$ is a cyclically reduced word lying in the subgroup of the free group on $c$ and $d$ generated by $c^{2}$ and the derived subgroup and there exists $l_{i}$ such that $\left|l_{i}\right| \geqslant 2$. This case has just been considered above.

Thus, let $R(a, b)=a b a b^{-1} \cdots a b a b^{-1}=\left(a b a b^{-1}\right)^{l}$ for some $l>0$. Then the group $G$ has a presentation of the following form:

$$
G=\left\langle a, b \mid a^{2}=\left(a b a b^{-1}\right)^{t}=1\right\rangle,
$$

where $t \geqslant 2$. We claim that there exist matrices $A, B \in \mathrm{SL}_{2}(\mathbb{C})$ satisfying the conditions
(1) $\operatorname{tr} A=0$;
(2) $\operatorname{tr} A B^{-2} A B^{3}=2$;
(3) $\operatorname{tr} A B \notin \mathcal{O}$;
(4) $\operatorname{tr} A B A B^{-1}=c$;

where $c= \pm 2 \cos (r \pi / m)$ for some $r \in\{1, \ldots, m-1\}$. Using Fricke characters one can write conditions (2) and (4) as the system

$$
\left\{\begin{array}{l}
\tau_{g h g h^{-1}}(A, B)=c \\
\tau_{g h^{-2} g h^{3}}(A, B)=2
\end{array}\right.
$$

Using relations (1) for Fricke characters it is easy to obtain

$$
\tau_{g h h^{-1}}=-\tau_{g h}^{2}+\tau_{g} \tau_{h} \tau_{g h}-\tau_{h}^{2}+2
$$

and

$$
\tau_{g h^{-2} g h^{3}}=\left(\tau_{g} \tau_{h}^{2}-\tau_{h} \tau_{g h}-\tau_{g}\right)\left(\tau_{h}^{2} \tau_{g h}-\tau_{g} \tau_{h}-\tau_{g h}\right)-\tau_{h}^{5}+5 \tau_{h}^{3}-5 \tau_{h} .
$$

We set $y=\tau_{h}(A, B)=\operatorname{tr} B$ and $z=\tau_{g h}(A, B)=\operatorname{tr} A B$. Since $\tau_{g}(A, B)=\operatorname{tr} A=0$, it follows that

$$
\tau_{g h g h^{-1}}(A, B)=-z^{2}-y^{2}+2, \quad \tau_{g h^{-2} g h^{3}}(A, B)=-\left(y^{3}-y\right) z^{2}-\left(y^{5}-5 y^{3}+5 y\right) .
$$

Thus, one can write (8) in the following form:

$$
\left\{\begin{array}{l}
z^{2}+y^{2}-2+c=0 \\
\left(y^{3}-y\right) z^{2}+y^{5}-5 y^{3}+5 y+2=0 .
\end{array}\right.
$$

It follows by (9) that

$$
y^{3}-\left(1+\frac{1}{c+2}\right) y-\frac{2}{c+2}=0 .
$$

By Lemma 5(2) there exists $r \in\{1, \ldots, m-1\}$ such that $1 /(c+2)$ does not belong to $\mathcal{O}$. In this case equation (10) has a root $y_{0} \notin \mathcal{O}$. Let $\left(y_{0}, z_{0}\right)$ be some solution of (9). By Lemma 2 there exist matrices $A, B \in \mathrm{SL}_{2}(\mathbb{C})$ such that

$$
\operatorname{tr} A=0, \quad \operatorname{tr} B=y_{0}, \quad \text { and } \quad \operatorname{tr} A B=z_{0} .
$$

Lemma 4 completes the proof of Theorem 2 in the second case. 
Case 3. Let $R(a, b)=a b^{n_{1}} \cdots a b^{n_{s}}$ and assume that $R(a, b)$ does not belong to the subgroup of the free group on $a$ and $b$ generated by $a^{2}$ and the derived subgroup. The case $\sum_{i=1}^{s} n_{i} \neq 0$ has been considered in [2], Theorem 5 . Hence we can assume that $s$ is odd and $\sum_{i=1}^{s} n_{i}=0$.

First, we consider the case $m=2$. Let $G_{1}=\left\langle a, b \mid a^{2}=b^{2}=R^{2}(a, b)=1\right\rangle$. We claim that $G_{1}=\left\langle a, b \mid a^{2}=b^{2}=1\right\rangle$ is the free product of two cyclic groups of order two. It is sufficient to prove that $R^{2}(a, b)=1$ in $G$. We shall prove a more general fact: if $w(a, b)=a b^{l_{1}} \cdots a b^{l_{s}}$, where $s=2 k+1$ and $\sum_{i=1}^{s} l_{s}$ is even, then $w^{2}(a, b)=1$ in $G_{1}$. We use induction on $s$. For $s=1$ the claim is obvious. For arbitrary $s$ there exists an even number among the exponents $l_{1}, \ldots, l_{s}$. Assume, for example, that $l_{1}$ is even. Then $b^{l_{1}}=1$ in $G_{1}$ and

$$
w(a, b)=b^{l_{2}} a b^{l_{3}} \cdots a b^{l_{s}},
$$

that is, $w(a, b)$ is conjugate to

$$
w_{1}(a, b)=a b^{l_{3}} \cdots a b^{l_{2}+l_{s}} .
$$

Since the sum $l_{3}+\cdots+\left(l_{2}+l_{s}\right)$ is even as before, we can use induction and obtain that $w_{1}^{2}(a, b)=1$ in $G_{1}$. Hence $w^{2}(a, b)=1$ in $G_{1}$. Since the group $G_{1}$ is an epimorphic image of $G$, it follows that $G$ is a non-trivial free product with amalgamation.

Next, we consider the case of $m \geqslant 3$. We claim that there exist matrices $A, B$ in $\mathrm{SL}_{2}(\mathbb{C})$ such that

$$
\operatorname{tr} A=0, \quad \operatorname{tr} B=y_{0}, \quad \operatorname{tr} A B=2, \quad \text { and } \quad Q_{R}\left(0, y_{0}, 2\right)=2 \cos \frac{\pi}{m},
$$

where $y_{0}$ is a complex number that is not an algebraic integer and $Q_{R}(x, y, z)$ is the Fricke polynomial of the word $R(g, h) \in F_{2}$. We have the following result.

Lemma 7. (1) $Q_{R}(0, y, z)=z f(y, z)$, where $f(y, z) \in \mathbb{Z}[y, z]$.

(2) The polynomial $f_{1}(y)=f(y, 2)$ is not constant.

Proof. (1) It follows from Lemma 3 that $Q_{g h^{n}}(0, y, z)=P_{n-1}(y) z$, therefore the assertion is proved for $s=1$. Assume that $s>1$. Then, using relations (1) one obtains

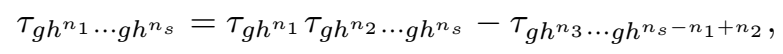

and by induction we have

$$
Q_{R}(0, y, z)=P_{n_{1}-1}(y) f_{1}(y, z) z-z f_{2}(y, z)=z f(y, z)
$$

for some polynomial $f(y, z) \in \mathbb{Z}[y, z]$.

(2) Consider the $\mathbb{Z}$-algebra $T$ generated by all Fricke characters $\tau_{w}, w \in F_{2}$. Each automorphism $\sigma \in \operatorname{Aut}\left(F_{2}\right)$ induces an automorphism

$$
\sigma^{\prime}: T \rightarrow T, \quad \tau_{w} \mapsto \tau_{\sigma(w)} .
$$


We consider an automorphism $\sigma \in \operatorname{Aut}\left(F_{2}\right)$ such that

$$
\sigma(g)=g, \quad \sigma(h)=g h .
$$

Then $\sigma^{\prime} \in \operatorname{Aut}(T)$ is an automorphism such that

$$
\sigma^{\prime}(x)=x_{1}=x, \quad \sigma^{\prime}(y)=y_{1}=z, \quad \sigma^{\prime}(z)=z_{1}=x z-y .
$$

Furthermore,

$$
\sigma^{\prime}\left(\tau_{R(g, h)}\right)=\tau_{\sigma(R(g, h))}=\tau_{R(\sigma(g), \sigma(h))},
$$

therefore

$$
\sigma^{\prime}\left(Q_{R}(x, y, z)\right)=Q_{R}\left(x_{1}, y_{1}, z_{1}\right)=Q_{R}(x, z, x z-y) .
$$

Thus, $\sigma^{\prime}\left(Q_{R}(0, y, 2)\right)=Q_{R}(0,2,-y)$. It follows from Proposition 2 that the polynomial $Q_{R}(0,2,-y) \in \mathbb{Z}[y]$ has degree $s$ because its leading coefficient $M_{s}$ satisfies the inequality $\left|M_{s}\right|=\prod_{i=1}^{s}\left|n_{i}\right| \neq 0$. Hence $Q_{R}(0,2,-y)$ is not constant, so that $\sigma^{\prime-1}\left(Q_{R}(0,2,-y)\right)=Q_{R}(0, y, 2)=2 f_{1}(y)$ is not constant either, which completes the proof.

We can now complete the proof of Theorem 1. By Lemma 7 the equality $Q_{R}\left(0, y_{0}, 2\right)=2 \cos (\pi / m)$ is equivalent to the relation

$$
f_{1}\left(y_{0}\right)=\cos \frac{\pi}{m} .
$$

By Lemma $5(3)$, the number $\cos (\pi / m)$ is not an algebraic integer, therefore there exists a root $y_{0}$ of equation (11) that is not an algebraic integer either. By Lemma 2 there exist matrices $A, B \in \mathrm{SL}_{2}(\mathbb{C})$ such that

$$
\operatorname{tr} A=0, \quad \operatorname{tr} B=y_{0}, \quad \text { and } \quad \operatorname{tr} A B=2 .
$$

Using Lemma 4 we complete the proof of Theorem 1.

\section{$\S 3$. Proof of Theorem 2}

Bearing in mind Theorem 1 we can assume that $n \geqslant 3$. Let $R(g, h) \in F_{2}$ and let $Q_{R}$ be the Fricke polynomial of the word $R$. We claim that there exist matrices $A, B \in \mathrm{SL}_{2}(\mathbb{C})$ such that

$$
\operatorname{tr} A=\alpha, \quad \operatorname{tr} B=2, \quad \text { and } \quad \operatorname{tr} A B=z,
$$

where $\alpha=2 \cos (\pi / n), z$ is a complex number that is not an algebraic integer and satisfies the equation

$$
Q_{R}(\alpha, 2, z)=c,
$$

where $c= \pm 2 \cos (r \pi / m)$ for some $r \in\{1, \ldots, m-1\}$. By Proposition 2 we can rewrite (12) in the following form:

$$
M_{s}(\alpha, 2) z^{s}+M_{s-1}(\alpha, 2) z^{s-1}+\cdots+M_{0}(\alpha, 2)-c=0,
$$


where

$$
M_{s}(\alpha, 2)=\prod_{i=1}^{s} P_{u_{i}-1}(\alpha) P_{v_{i}-1}(2) .
$$

By Lemma $3, P_{u_{i}-1}(\alpha) \neq 0$ and $P_{v_{i}-1}(2)=v_{i}$, therefore $M_{s}(\alpha, 2) \neq 0$. We write (13) in the following form:

$$
z^{s}+\frac{M_{s-1}(\alpha, 2)}{M_{s}(\alpha, 2)}+\cdots+\frac{M_{0}(\alpha, 2)-c}{M_{s}(\alpha, 2)}=0 .
$$

We claim that we can choose $c$ such that $\left(M_{0}(\alpha, 2)-c\right) / M_{s}(\alpha, 2) \notin \mathcal{O}$. Assume that both quantities

$$
\frac{M_{0}(\alpha, 2)+2 \cos (r \pi / m)}{M_{s}(\alpha, 2)} \quad \text { and } \quad \frac{M_{0}(\alpha, 2)-2 \cos (r \pi / m)}{M_{s}(\alpha, 2)}
$$

belong to $\mathcal{O}$ for each $r \in\{1, \ldots, m-1\}$. Then, for their difference we have

$$
\frac{4 \cos (r \pi / m)}{M_{s}(\alpha, 2)} \in \mathcal{O}
$$

Since $P_{r}(\lambda)$ has integer coefficients for each $r$, it follows that $P_{u_{i}-1}(\alpha)$ belongs to $\mathcal{O}$. Hence

$$
\frac{4 \cos (r \pi / m)}{M_{s}(\alpha, 2)} P_{u_{i}-1}(\alpha)=\frac{4 \cos (r \pi / m)}{M} \in \mathcal{O}
$$

for each $r \in\{1, \ldots, m-1\}$, where $M=\prod_{i=1}^{s} v_{i} \in \mathbb{Z}$ and $|M| \geqslant 3$ by the assumptions of the theorem. We obtain a contradiction with Lemma 5(4). This means that there exists a root $z_{0}$ of the equation (14) that does not belong to $\mathcal{O}$. By Lemma 2 there exist matrices $A, B \in \mathrm{SL}_{2}(\mathbb{C})$ such that

$$
\operatorname{tr} A=\alpha, \quad \operatorname{tr} B=2, \quad \text { and } \quad \operatorname{tr} A B=z_{0} .
$$

Using Lemma 4 we complete the proof of Theorem 2.

In conclusion, we state the following conjecture.

Conjecture 2. The group $G=\left\langle a, b \mid a^{n}=R^{m}(a, b)=1\right\rangle$, where $m \geqslant 2, n=0$ or $n \geqslant 2$, and $R(a, b)$ is a cyclically reduced word containing $b$ in the free group on a and $b$, is a non-trivial free product with amalgamation.

In view of Theorems 1, 2 and [2], Theorem 5 , this conjecture has not yet been proved in the following case: $n$ is odd and $R(a, b)=a^{l} R_{1}(a, b)$, where $0 \leqslant l<n$ and $R_{1}(a, b)=a^{u_{1}} b^{v_{1}} \cdots a^{u_{s}} b^{v_{s}}$ is a cyclically reduced word belonging to the derived subgroup of the free group on $a$ and $b$ such that $\prod_{i=1}^{s}\left|v_{i}\right| \in\{1,2\}$.

This paper was written while the author was visiting the University of Bielefeld, Germany as a guest of SFB 343 "Diskrete Strukturen in der Mathematik". 


\section{Bibliography}

[1] J. Howie, "One-relator products of groups", Groups (St. Andrews 1985), Cambridge Univ. Press, Cambridge 1986, pp. 216-220.

[2] B. Fine, F. Levin, and G. Rosenberger, "Free subgroups and decompositions of one-relator products of cyclics, Part 2: Normal torsion-free subgroups and FPA decompositions", J. Indian Math. Soc. 49 (1985), 237-247.

[3] H. Zieschang, "On decompositions of discontinuous groups of the plane", Math. Z. 151 (1976), 165-188.

[4] G. Rosenberger, "Bemerkungen zu einer Arbeit von H. Zieschang", Arch. Math. (Basel) 29 (1977), 623-627.

[5] D. D. Long, C. Maclachlan, and A. W. Reid, "Splitting groups of signature (1, n)", J. Algebra 185 (1996), 329-341.

[6] M. J. Dunwoody and M. Sageev, "Splittings of certain Fuchsian groups", Proc. Amer. Math. Soc. 125 (1997), 1953-1954.

[7] W. Magnus, A. Karrass, and D. Solitar, Combinatorial group theory. Presentation of groups in terms of generators and relations, Interscience, New York 1974.

[8] H. Bass, "Finitely generated subgroups of $\mathrm{GL}_{2}(\mathbb{C})_{2}(\mathbb{C})$ ", The Smith Conjecture, Wiley, New York 1984, pp. 127-136.

[9] R. Horowitz, "Characters of free groups represented in the two-dimensional linear group", Comm. Pure Appl. Math. 25 (1972), 635-649.

[10] W. Magnus, "The uses of 2 by 2 matrices in combinatorial group theory", Results Math. 4:2 (1981), 171-192.

[11] M. Culler and P. Shalen, "Varieties of group representations and splittings of 3 manifolds", Ann. of Math. (2) 117 (1983), 109-147.

[12] H. Helling, "Diskrete Untergruppen von $\mathrm{SL}_{2}(\mathbb{R})$ ", Invent. Math. 17 (1972), 217-229.

[13] C. Traina, "Trace polynomial for two generated subgroups of $\mathrm{SL}_{2}(\mathbb{C})$ ", Proc. Amer. Math. Soc. 79 (1980), 369-372.

Institute of Mathematics

National Academy of Sciences of Belarus

Minsk

E-mail address: benyash@im.bas-net.by 